\title{
COMPOUND CHANNELS, TRANSITION EXPECTATIONS AND LIFTINGS
}

\author{
L. Accardi \\ Dipartimento di Matematica \\ Centro Matematico V.Volterra \\ Universitá di Roma II, Italy
}

\author{
M. Ohya \\ Department of \\ Information Sciences \\ Science University of Tokyo, Japan
}

\begin{abstract}
.
In Section 1, we introduce the notion of lifting as a generalization of the notion of compound state introduced in [21], [22] and we show that this notion allows an unified approach to the problems of quantum measurement and of signal transmission through quantum channels. The dual of a linear lifting is a transition expectation in the sense of [3] and we characterize those transition expectations which arise from compound states in the sense of [22].

In Section 2, we characterize those liftings whose range is contained in the closed convex hull of product states and we prove that the corresponding quantum Markov chains [2] are uniquely determined by a classical generalization of both the quantum random walks of [4] and the locally diagonalizable states considered in [3].

In Section 4, as a first application of the above results, we prove that the attenuation (beam splitting) process for optical communication treated in [21] can be described in a simpler and more general way in terms of liftings and of transition expectations. The error probabilty of information transmission in the attenuation process is rederived from our new description. We also obtain some new results concerning the explicit computation of error probabilities in the squeezing case.
\end{abstract}

\section{Key Words.}

Compound State, Transition Expectation, Lifting, Channel, Quantum Probability, Quantum Markov Chain, Beam Splitting, Optical Communication

\section{AMS Classification. \\ 81Q99, 94A40, 60J27}

\section{Introduction}

The following situation is very common both in classical and quantum physics: one considers two systems, denoted respectively 1, 2 and their algebras of observables, $\mathcal{A}_{1}, \mathcal{A}_{2}$. One usually assumes that the interaction between the two systems is switched on at a sharp time $t_{o}$ before which the two systems are considered to be independent. During 
the interaction the two systems merge into a larger system denoted $(1,2)$ whose algebra of observables $\mathcal{A}$ contains both $\mathcal{A}_{1}$ and $\mathcal{A}_{2}$, in the sense that there are embeddings

$$
j_{1}: \mathcal{A}_{1} \rightarrow \mathcal{A} ; j_{2}: \mathcal{A}_{2} \rightarrow \mathcal{A}
$$

and that any physical information on the state of system 1 or of system 2 after the interaction can be obtained by choosing a state $\varphi$ on $\mathcal{A}$, i.e. a state of the composite system $(1,2)$, and considering its restriction on the algebra $j_{1}\left(\mathcal{A}_{1}\right)\left(\operatorname{resp} . j_{2}\left(\mathcal{A}_{2}\right)\right)$. In most applications one chooses

$$
\begin{aligned}
\mathcal{A} & =\mathcal{A}_{1} \otimes \mathcal{A}_{2} ; \varphi=\varphi_{1} \otimes \varphi_{2} \\
j_{1}\left(a_{1}\right) & =a_{1} \otimes 1_{2} ; j_{2}\left(a_{2}\right)=1_{1} \otimes a_{2}, \quad a_{1} \in \mathcal{A}_{1}, a_{2} \in \mathcal{A}_{2}
\end{aligned}
$$

(i.e.,) the algebra of the compound system is described by a tensor product. In the present paper we shall confine our analysis to such a situation.

The choice of the state $\varphi$ depends on the initial states of the two systems and on the interaction between them. In connection with this situation one studies several problems depending on the interpretation of the systems 1 and 2. For example:

i) the state $\varphi_{2}$ of system 2 , after the interaction, is known (e.g., an output signal, a pointer in a measurement apparatus) and one wants to know the state $\varphi_{1}$ of system 1 before the interaction (e.g., an input signal, the state of a microsystem which has interacted with the apparatus).

ii) as in (i), exchanging the roles of 1 and 2. ¿From the mathematical point of view, this exchange is trivial, but we want to underline that our approach avoids the separation of a macroworld, described by classical physics, from a microworld, described by quantum physics.

iii) the initial state of the composite system $(1,2)$ is known and one wants to know the state of system 1 (system 2 ).

iv) the state $\varphi_{1}$ of system 1 , before the interaction (e.g., the preparation of a microsystem) and the form of the interaction, are known and one wants to know the state of system 1 after the interaction.

In all these cases the goal is to construct a map from the state space of a system to the state space of another system. In the literature on quantum information and communication systems, such a map is called a channel [20]. An important class of channels are those from the state space of an algebra $\mathcal{A}_{1}$ into the state space of the algebra $\mathcal{A}_{1} \otimes \mathcal{A}_{2}$. This channels are called liftings; more generally, a lifting should be through as a channel from a sub-system to a compound system. An important example of liftings are the duals of transition expectations.

1. \%par

Recall (cf. Definition (1.3) below) that if $\mathcal{A}_{1}, \mathcal{A}_{2}$ are $\mathrm{C}^{*}$-algebras, a transition expectation from $\mathcal{A}_{1} \otimes \mathcal{A}_{2}$ to $\mathcal{A}_{1}$ is a completely positive linear map $\mathcal{E}: \mathcal{A}_{1} \otimes \mathcal{A}_{2} \rightarrow \mathcal{A}_{1}$ satisfying (1.6).

Transition expectations play a crucial role in the construction of quantum Markov chains and they arise naturally within the framework of measurement theory in the 
following way: the composite system $(1,2)$ undergoes an evolution $u_{t}: \mathcal{A} \rightarrow \mathcal{A}(t \in R)$, which is a one-parameter group of $*$-automorphisms of $\mathcal{A}$. This means that the state $\varphi$ of $(1,2)$ evolves according to the law

$$
\varphi_{t}:=\varphi \circ u_{t}
$$

and the state $\varphi_{1}$ of the system 1 evolves according to the reduced evolution:

$$
\varphi_{1, t}\left(a_{1}\right):=\varphi_{1}\left(E_{2} \circ u_{t} \circ j_{1}\left(a_{1}\right)\right) ; a_{1} \in \mathcal{A}_{\infty}
$$

where $j_{1}$ is given by $(0.3)$ and $E_{2}: \mathcal{A}=\mathcal{A}_{1} \otimes \mathcal{A}_{2} \rightarrow \mathcal{A}_{1}$ is the Umegaki conditional expectation characterized by

$$
E_{2}\left(a_{1} \otimes a_{2}\right)=a_{1} \varphi_{2}\left(a_{2}\right) ; a_{1} \in \mathcal{A}_{1} \quad ; a_{2} \in \mathcal{A}_{2}
$$

Let us fix a time $T$ representing the moment when the experiment ends (ideally $T=+\infty$ ) and consider the linear map $\mathcal{E}_{T}: \mathcal{A}_{1} \otimes \mathcal{A}_{2} \rightarrow \mathcal{A}_{1}$ characterized by

$$
\mathcal{E}_{T}\left(a_{1} \otimes a_{2}\right)=E_{2}\left(u_{T}\left(a_{1} \otimes a_{2}\right)\right) ; a_{1} \in \mathcal{A}_{1} ; a_{2} \in \mathcal{A}_{2}
$$

Then $\mathcal{E}_{T}$ is a transition expectation. If one is ready to accept that the evolution $u_{T}$ does not take place inside the algbra $\mathcal{A}_{1} \otimes \mathcal{A}_{2}$ but is a representation of $\mathcal{A}_{1} \otimes \mathcal{A}_{2}$ into another algebra (usually much larger), then in some cases and in a certain technical sense, (0.7) represents the most general class of transition expectations (cf. Theorem (1.4) below).

An instrument in the sense of the operational approach to quantum measurment is obtained by taking the restriction of a transition expectation $\mathcal{E}$ to a subalgebra $\mathcal{C}_{1} \otimes \mathcal{A}_{2}$ of $\mathcal{A}_{1} \otimes \mathcal{A}_{2}$ where $\mathcal{C}_{1}$ is a $\sigma$-finite abelian von Neumann sup-algebra of $\mathcal{A}_{1}$. In this case it is known that, if $\mathcal{C}_{1}$ is $\sigma$-finite, then there exists a probability space $(\Omega, \mathcal{F}, P)$ such that $\mathcal{C}_{1}$ is isomorphic to $L^{\infty}(\Omega, \mathcal{F}, P)$ and the points $\omega \in \Omega$ are interpreted as macroscopic parameters of the apparatus. If $\mathcal{A}_{1}=\mathcal{C}_{1}$, i.e. if $\mathcal{A}_{1}$ is an abelian von Neumann algebra, the isomorphism (cf. [28])

$$
\mathcal{A}=\mathcal{A}_{1} \otimes \mathcal{A}_{2}=L^{\infty}(\Omega, \mathcal{F}, P) \otimes \mathcal{A}_{2} \cong L^{\infty}\left(\Omega, \mathcal{F}, P ; \mathcal{A}_{2}\right)
$$

implies that the elements of $\mathcal{A}$, i.e., the observables of the composite system $(1,2)$ can be interpreted as functions $(\Omega, \mathcal{F}, P) \rightarrow \mathcal{A}_{2}$, i.e., as operator valued random variables. Thus interpreting $(\Omega, \mathcal{F}, P)$ as the sample space of a classical stochastic process, the operational scheme becomes equivalent to the theory of operator valued classical processes (cf. [7]).

¿From this point of view an instrument in the operational sense is an object which is only half-quantum. The physical motivations for this choice go back to some ideas of Ludwig according to which the measurement apparatus is usually a macroscopic body so that classical probability should be sufficient for its description. Several authors have introduced variations and modifications of Ludwig ideas, however, since all the 
examples of physical interest of instruments in operational sense, produced up to now, are the restrictions of liftings, we feel that the latter notion plays a more natural and fundamental role.

In conclusion of the present introduction, we show the theory of lifting includes the so calld operational approach.

\section{CHANNELS, LIFTINGS AND TRANSITION EXPECTATIONS}

For a $\mathrm{C}^{*}$-algebra $\mathcal{A}$, we denote $\mathcal{S}(\mathcal{A})$ the convex set of its states. In this paper all $\mathrm{C}^{*}$ - and $\mathrm{W}^{*}$-algebras are realized on some separable Hilbert spaces and, unless explicitly stated, the tensor products are those induced by the tensor products of the corresponding Hilbert spaces. If $\mathcal{A}$ is a von Neumann algebra, $\mathcal{S}(\mathcal{A})$ denotes the set of its normal states and $\mathcal{S}(\mathcal{A})_{\text {extr }}$ the set of extremal states. Both $\mathcal{S}(\mathcal{A})$ and $\mathcal{S}(\mathcal{A})_{\text {extr }}$ are measurable spaces with their Borel structure and the set of probability measures on $\mathcal{S}(\mathcal{A})\left(\mathcal{S}(\mathcal{A})_{\text {extr }}\right)$ is denoted $\operatorname{ProbS}(\mathcal{A})\left(\operatorname{ProbS}(\mathcal{A})_{\text {extr }}\right)$. If $\mathcal{A}$ and $\mathcal{B}$ are $\mathrm{C}^{*}$-algebras, a channel from $\mathcal{A}$ to $\mathcal{B}$ is a map $\Lambda^{*}: \mathcal{S}(\mathcal{A}) \rightarrow \mathcal{S}(\mathcal{B})$. If $\Lambda^{*}$ is affine we speak of a linear channel . If $\Lambda^{*}$ is $w *$-continuous and linear, then it can be extended by linearity to a linear map (still denoted $\Lambda^{*}$ ) from $\mathcal{A}^{*}$ to $\mathcal{B}^{*}$. Its dual $\Lambda: \mathcal{B} \rightarrow \mathcal{A}$ is a positive map. If it is completely positive, we call it a Markovian operator. Such channels have been studied with some applications by several authors (c.f., [20], [25] and references quoted theirs). Certain quantum channels are naturally associated to classical Markovian kernels on the measurable space $\mathcal{S}(\mathcal{B}) \times \mathcal{S}(\mathcal{A})$. In fact, given such a Markovian kernel, i.e., a measurable map

$$
p: \omega \in \mathcal{S}(\mathcal{A}) \longrightarrow p(\cdot \mid \omega) \in \operatorname{Prob}(\mathcal{S}(\mathcal{B}))
$$

one can define a channel in the following way: for any state $\varphi \in \mathcal{S}(\mathcal{A})$ one fixes a convex decomposition

$$
\varphi=\int_{\mathcal{S}(\mathcal{A})} \omega d \mu_{\varphi}(\omega)
$$

and defines the channel $\Lambda^{*}: \mathcal{S}(\mathcal{A}) \rightarrow \mathcal{S}(\mathcal{B})$ through the identity

$$
\Lambda^{*} \varphi:=\int_{\mathcal{S}(\mathcal{A})} d \mu_{\varphi}(\omega) \int_{\mathcal{S}(\mathcal{B})} \omega^{\prime} p\left(d \omega^{\prime} \mid \omega\right)
$$

The channel $\Lambda^{*}$ is usually nonlinear since the map $\varphi \rightarrow \mu_{\varphi}$ is affine if and only if $\mathcal{S}(\mathcal{A})$ is a simplex ([8] Theorem (4.1.15) and Corollary (4.1.17)) and this is the case if and only if $\mathcal{A}$ is Abelian ([8] Example (4.1.6) ). On the other hand, given a linear channel $\Lambda^{*}$ one might try to associate to it a Markovian kernel $p(\cdot \mid \omega)$ on the measurable space $\mathcal{S}(\mathcal{B}) \times \mathcal{S}(\mathcal{A})$, by fixing, for each $\omega \in \mathcal{S}(\mathcal{A})$, a convex decomposition

$$
\Lambda^{*} \omega:=\int_{\mathcal{S}(\mathcal{B})} \omega^{\prime} p\left(d \omega^{\prime} \mid \omega\right)
$$

The possibility of choosing such a decomposition so to assure the measurability of the map

$$
p: \omega \in \mathcal{S}(\mathcal{A}) \longrightarrow p(\cdot \mid \omega) \in \operatorname{Prob}(\mathcal{S}(\mathcal{B}))
$$


as well as the study of the support of these measures give rise to some subtle measure theoretic problems which will be discussed elsewhere. In many examples however, these Markovian kernels can be explicitly constructed and, at least on a subset of the states and have good support and measurability properties.

Definition 1.1: Let $\mathcal{A}_{1}, \mathcal{A}_{2}$ be $\mathrm{C}^{*}$-algebras and let $\mathcal{A}_{1} \otimes \mathcal{A}_{2}$ be a fixed $\mathrm{C}^{*}$-tensor product of $\mathcal{A}_{1}$ and $\mathcal{A}_{2}$. A lifting from $\mathcal{A}_{1}$ to $\mathcal{A}_{1} \otimes \mathcal{A}_{2}$ is a $w *$-continuous map

$$
\mathcal{E}^{*}: \mathcal{S}\left(\mathcal{A}_{1}\right) \rightarrow \mathcal{S}\left(\mathcal{A}_{1} \otimes \mathcal{A}_{2}\right)
$$

If $\mathcal{E}^{*}$ is affine and its dual is a completely positive map, we call it a linear lifting; if it maps pure states into pure states, we call it pure.

Remark: Also in the nonlinear case some kinds of complete positivity requirement should be included in the definition of lifting. However, the theory of nonlinear completely positive maps is still in its infancy and the some is true for a satisfactory dualization of it. Therefore we leave the general question open for further developments and we limit ourselves to present some examples of nonlinear liftings which are of some interest for the applications.

To every lifting from $\mathcal{A}_{1}$ to $\mathcal{A}_{1} \otimes \mathcal{A}_{2}$ we can associate two channels: one from $\mathcal{A}_{1}$ to $\mathcal{A}_{1}$ , defined by

$$
\Lambda_{1}^{*} \rho_{1}\left(a_{1}\right):=\left(\mathcal{E}^{*} \rho_{1}\right)\left(a_{1} \otimes 1\right) \quad ; \quad \forall a_{1} \in \mathcal{A}_{1}
$$

another from $\mathcal{A}_{1}$ to $\mathcal{A}_{2}$, defined by

$$
\Lambda_{2}^{*} \rho_{1}\left(a_{2}\right):=\left(\mathcal{E}^{*} \rho_{1}\right)\left(1 \otimes a_{2}\right) \quad ; \quad \forall a_{2} \in \mathcal{A}_{2}
$$

In general, a state $\varphi \in \mathcal{S}\left(\mathcal{A}_{1} \otimes \mathcal{A}_{2}\right)$ such that

$$
\left.\varphi\right|_{\mathcal{A}_{1} \otimes 1}=\rho_{1} \quad ;\left.\quad \varphi\right|_{1 \otimes \mathcal{A}_{2}}=\rho_{2}
$$

has been called [21], [23] a compound state of the states $\rho_{1} \in \mathcal{S}\left(\mathcal{A}_{1}\right)$ and $\rho_{2} \in \mathcal{S}\left(\mathcal{A}_{2}\right)$ . In classical probability theory, also the term coupling between $\rho_{1}$ and $\rho_{2}$ is used [13].

The following problem is important in several applications: Given a state $\rho_{1} \in \mathcal{S}\left(\mathcal{A}_{1}\right)$ and a channel $\Lambda^{*}: \mathcal{S}\left(\mathcal{A}_{1}\right) \rightarrow \mathcal{S}\left(\mathcal{A}_{2}\right)$, find a standard lifting $\mathcal{E}^{*}: \mathcal{S}\left(\mathcal{A}_{1}\right) \rightarrow \mathcal{S}\left(\mathcal{A}_{1} \otimes \mathcal{A}_{2}\right)$ such that $\mathcal{E}^{*} \rho_{1}$ is a compound state of $\rho_{1}$ and $\Lambda^{*} \rho_{1}$. Several particular solutions of this problem have been proposed in [9], [10], [11], [21], [22], [23], however an explicit description of all the possible solutions to this problem is still missing.

Definition 1.2: A lifting from $\mathcal{A}_{1}$ to $\mathcal{A}_{1} \otimes \mathcal{A}_{2}$ is called nondemolition for a state $\rho_{1} \in \mathcal{S}\left(\mathcal{A}_{1}\right)$ if $\rho_{1}$ is invariant for $\Lambda_{1}^{*}$ i.e., if for all $a_{1} \in \mathcal{A}_{1}$

$$
\left(\mathcal{E}^{*} \rho_{1}\right)\left(a_{1} \otimes 1\right)=\rho_{1}\left(a_{1}\right)
$$


The idea of this definition being that the interaction with system 2 does not alter the state of system 1 .

Definition 1.3: Let $\mathcal{A}_{1}, \mathcal{A}_{2}$ be $\mathrm{C}^{*}$-algebras and let $\mathcal{A}_{1} \otimes \mathcal{A}_{2}$ be a fixed $\mathrm{C}^{*}$-tensor product of $\mathcal{A}_{1}$ and $\mathcal{A}_{2}$. A transition expectation from $\mathcal{A}_{1} \otimes \mathcal{A}_{2}$ to $\mathcal{A}_{1}$ is a completely positive linear map $\mathcal{E}: \mathcal{A}_{1} \otimes \mathcal{A}_{2} \rightarrow \mathcal{A}_{1}$ satisfying

$$
\mathcal{E}\left(1_{\mathcal{A}_{1}} \otimes 1_{\mathcal{A}_{2}}\right)=1_{\mathcal{A}_{1}} .
$$

Remark: The notion of nondemolition lifting, discussed here is essentially (i.e., up to minor technicalities) included in the more abstract notion of state extension introduced by Cecchini and Petz [10], [11] (cf. also Cecchini and Kümmerer [11]).

The two interpretations of the notion of standard lifting, which shall be used in the present paper, are the following:

\section{(1) The measurement process}

$\mathcal{A}_{1}$ (resp. $\mathcal{A}_{2}$ ) is interpreted as the algebra of observables of a system (resp. a measurement apparatus ) and $\mathcal{E}^{*}$ describes the interaction between system and apparatus as well as the preparation of the apparatus. If $\rho_{1} \in \mathcal{S}\left(\mathcal{A}_{1}\right)$ is the preparation of the system, i.e., its state before the interaction with the apparatus, then $\Lambda_{1}^{*} \rho_{1} \in \mathcal{S}\left(\mathcal{A}_{1}\right)$ (resp. $\Lambda_{2}^{*} \rho_{1} \in \mathcal{S}\left(\mathcal{A}_{2}\right)$ ) is the state of the system (resp. of the apparatus) after the measurement.

\section{(2) The signal transmission process}

An input signal is transmitted and received by an apparatus which produces an output signal. Here $\mathcal{A}_{1}$ (resp. $\mathcal{A}_{2}$ ) is interpreted as the algebra of observables of the input (resp. output) signal and $\mathcal{E}^{*}$ describes the interaction between the input signal and the receiver as well as the preparation of the receiver. If $\rho_{1} \in \mathcal{S}\left(\mathcal{A}_{1}\right)$ is the input signal, then the state $\Lambda_{2}^{*} \rho_{1} \in \mathcal{S}\left(\mathcal{A}_{2}\right)$, defined by (1.3) is the state of the (observed) output signal.

An important lifting related to this signal transmission is one due to a quantum communication process discussed below (Example 1a and 4).

In several important applications the state $\rho_{1}$ of the system before the interaction (preparation, input signal) is not known and one would like to know this state knowing only $\Lambda_{2}^{*} \rho_{1} \in \mathcal{S}\left(\mathcal{A}_{2}\right)$, i.e., the state of the apparatus after the interaction (output signal). From a mathematical point of view this problem is not well posed, since usually the map $\Lambda_{2}^{*}$ is not invertible. The best one can do in such cases is to acquire a control on the description of those input states which have the same image under $\Lambda_{2}^{*}$ and then choose among them according to some statistical criterion. Another widely applied procedure is to postulate, on the basis of some experimental information, that the input state belongs to an a priori given restricted class of states and to choose among these ones by some statistical criterion. 
In the following we describe several examples of liftings which appear frequently in the applications.

Example 1: Isometric liftings.

Let $V: \mathcal{H}_{1} \rightarrow \mathcal{H}_{1} \otimes \mathcal{H}_{2}$ be an isometry

$$
V^{*} V=1_{\mathcal{H}_{1}}
$$

Then the map

$$
\mathcal{E}: x \in \mathcal{B}\left(\mathcal{H}_{1}\right) \otimes \mathcal{B}\left(\mathcal{H}_{2}\right) \rightarrow V^{*} x V \in \mathcal{B}\left(\mathcal{H}_{1}\right)
$$

is a transition expectation, and the associated lifting maps a density matrix $w_{1} \in T\left(\mathcal{H}_{1}\right)$ into $\mathcal{E}^{*} w_{1}=V w_{1} V^{*}$. Liftings of this type are called isometric. Every isometric lifting is a pure lifting. Isometric liftings have turned out to play a relevant role in some mathematical models for superconductivity [14].

Example 1a: The attenuation (or beam splitting) lifting.

It is the particular isometric lifting characterized by the properties.

$$
\begin{gathered}
\mathcal{H}_{1}=\mathcal{H}_{2}=: \Gamma(\mathbf{C})=\text { the Fock space over } \mathbf{C} \\
V: \Gamma(\mathbf{C}) \rightarrow \Gamma(\mathbf{C}) \otimes \Gamma(\mathbf{C})
\end{gathered}
$$

is characterized by the expression

$$
V|\theta>=| \alpha \theta>\otimes \mid \beta \theta>
$$

where $\mid \theta>$ is the normalized coherent vector parametrized by $\theta \in \mathbf{C}$ and $\alpha, \beta \in \mathbf{C}$ are such that

$$
|\alpha|^{2}+|\beta|^{2}=1
$$

Notice that this liftings maps coherent states into products of coherent states. So it maps the simplex of the so called classical states (i.e., the convex combinations of coherent vectors) into itself. Restricted to these states it is of convex product type in the sense of Definition 2.1 below, but it is not of convex product type on the set of all states.

Denoting, for $\theta \in \mathbf{C}, \omega_{\theta}$ the state on $\mathcal{B}(\Gamma(\mathbf{C}))$ defined by

$$
\omega_{\theta}(b)=<\theta, b \theta>\quad ; b \in \mathcal{B}(\Gamma(\mathbf{C}))
$$

we see that, for any $b \in \mathcal{B}(\Gamma(\mathbf{C}))$

$$
\left(\mathcal{E}^{*} \omega_{\theta}\right)(b \otimes 1)=\omega_{\alpha \theta}(b)
$$

hence this lifting is not nondemolition.

Interpretation: $\Gamma(\mathbf{C})$ is the space of a 1-mode $E M$ field (signal). $V$ represents the interaction, of the signal with an apparatus (e.g., a receiver or a semi-transparent mirror). In $\Gamma(\mathbf{C}) \otimes \Gamma(\mathbf{C})$ the second factor is the space of the apparatus. 
Equation (1.10) means that, by the effect of the interaction, a coherent signal (beam) $\mid \theta>$ splits into 2 signals (beams) still coherent, but of lower intensity. Because of (1.11), the total intensity (energy) is preserved by the transformation.

\section{Example 1b: Superposition beam splitting.}

The only difference with Example (1a.) is the form of $V$, which in this case is

$$
V \mid \theta>=\frac{1}{\sqrt{2}}(|\alpha \theta>\otimes| \beta \theta>-i|\beta \theta>\otimes| \alpha \theta>)
$$

One easily checks that $V$ extends linearly to an isometry of the form (1.9).

This isometric lifting is not of convex product type in the sense of Definition 2.1 of the next section, neither it is a nondemolition lifting.

\section{Example 2: The compound lifting.}

Let $\Lambda^{*}: \mathcal{S}\left(\mathcal{A}_{1}\right) \rightarrow \mathcal{S}\left(\mathcal{A}_{2}\right)$ be a channel. For any $\rho_{1} \in \mathcal{S}\left(\mathcal{A}_{2}\right)$ in the closed convex hull of the external states, fix a decomposition of $\rho_{1}$ as a convex combination of extremal states in $\mathcal{S}\left(\mathcal{A}_{1}\right)$

$$
\rho_{1}=\int_{\mathcal{S}\left(\mathcal{A}_{1}\right)} \omega_{1} p\left(d \omega_{1} \mid \rho_{1}\right)
$$

where $p\left(\cdot \mid \rho_{1}\right)$ is a Borel measure on $\mathcal{S}\left(\mathcal{A}_{1}\right)$ with support in the extremal states, and define

$$
\mathcal{E}^{*} \rho_{1}:=\int_{\mathcal{S}\left(\mathcal{A}_{1}\right)} \omega_{1} \otimes \Lambda^{*} \omega_{1} p\left(d \omega_{1} \mid \rho_{1}\right)
$$

Then $\mathcal{E}^{*}: \mathcal{S}\left(\mathcal{A}_{1}\right) \rightarrow \mathcal{S}\left(\mathcal{A}_{2} \otimes \mathcal{A}_{2}\right)$ is a lifting, nonlinear even if $\Lambda^{*}$ is linear, and nondemolition for $\rho_{1}$. In Section 2, we shall see that the most general lifting, mapping $\mathcal{S}\left(\mathcal{A}_{1}\right)$ into the closed convex hull of the external product states on $\mathcal{A}_{1} \otimes \mathcal{A}_{2}$ is essentially of this type. Here "essentially" means that, in order to recover the most general case, we shall weaken, from the original definition of compound state in [22], the condition that $p\left(d \omega_{1} \mid \rho_{1}\right)$ is concentrated on the extremal states.

Therefore once a channel is given, a lifting of convex product type can be constructed by (1.16), and the converse is also true due to (1.3):

\section{channel $\longleftrightarrow$ lifting.}

For example, the von Neumann quantum measurement process is written, in our terminology, as follows: Having measured an observable $A=\sum_{n} a_{n} P_{n}$ (spectral decomposition with discrete spectrum) in a state $\rho$, the state after this measurement will be

$$
\Lambda^{*} \rho=\sum_{n} P_{n} \rho P_{n}
$$

and a lifting $\mathcal{E}^{*}$, of convex product type, associated to this channel $\Lambda^{*}$ and to a fixed decomposition of $\rho$ as $\rho=\sum_{n} \lambda_{n} \rho_{n}\left(\rho_{n} \in \mathcal{S}\left(\mathcal{A}_{1}\right)\right)$ is given by :

$$
\mathcal{E}^{*} \rho=\sum_{n} \lambda_{n} \rho_{n} \otimes \Lambda^{*} \rho_{n}
$$


A more sophisticated example of lifting of this type is a reduction of a state associated with an open system dynamics. Namely, if a system $\Sigma_{1}$ described by a Hilbert space $\mathcal{H}$ interacts with an external system $\Sigma_{2}$ described by another Hilbert space $\mathcal{K}$ and the initial states of $\Sigma_{1}$ and $\Sigma_{2}$ are $\rho$ and $\sigma$, respectively, then the combined state $\theta_{t}$ of $\Sigma_{1}$ and $\Sigma_{2}$ at time $t$ after the interaction between the two systems is given by

$$
\theta_{t}=U_{t}^{*}(\rho \otimes \sigma) U_{t}
$$

where $U_{t}=\exp (i t H)$ with the total Hamiltonian $H$ of $\Sigma_{1}$ and $\Sigma_{2}$. A channel is obtained by taking the partial trace with respect to $\mathcal{K}$ i.e.,

$$
\rho \rightarrow \Lambda_{t}^{*} \rho=\operatorname{tr}_{\mathcal{K}} \theta_{t} .
$$

A lifting associated to this channel is given by (1.17) with $\Lambda_{t}^{*}$ above.

\section{Example 3: Canonical form of a lifting}

Let $\mathcal{A}_{1}=\mathcal{B}\left(\mathcal{H}_{1}\right), \mathcal{A}_{2}=\mathcal{B}\left(\mathcal{H}_{2}\right)$. The most general linear lifting $\mathcal{E}^{*}: \mathcal{S}\left(\mathcal{A}_{1}\right) \rightarrow$ $\mathcal{S}\left(\mathcal{A}_{1} \otimes \mathcal{A}_{2}\right)$ has the form

$$
w_{1} \in T\left(\mathcal{H}_{1}\right) \rightarrow \sum K_{i}\left(w_{1} \otimes 1\right) K_{i}^{*} \in T\left(\mathcal{H}_{1} \otimes \mathcal{H}_{2}\right)
$$

for some $K_{i} \in \mathcal{B}\left(\mathcal{H}_{1} \otimes \mathcal{H}_{2}\right)$ such that

$$
\sum K_{i}^{*} K_{i}=1
$$

This is a simple consequence of Krein's Lemma.

Example 4: The lifting for quantum communication channel.

Let $\mathcal{H}_{1}, \mathcal{H}_{2}, \mathcal{K}_{1}, \mathcal{K}_{2}$ be Hilbert spaces. Denote $\alpha$ the amplification

$$
\alpha: b_{2} \in \mathcal{B}\left(\mathcal{H}_{2}\right) \rightarrow \alpha\left(b_{2}\right)=b_{2} \otimes 1_{\mathcal{K}_{2}} \in \mathcal{B}\left(\mathcal{H}_{2} \otimes \mathcal{K}_{2}\right)
$$

Let

$$
\gamma: \mathcal{B}\left(\mathcal{H}_{2} \otimes \mathcal{K}_{2}\right) \rightarrow \mathcal{B}\left(\mathcal{H}_{1} \otimes \mathcal{K}_{1}\right)
$$

be a completely positive identity preserving map and, for $\sigma_{1} \in \mathcal{S}\left(\mathcal{B}\left(\mathcal{K}_{1}\right)\right)$ denote $\bar{\sigma}_{1}^{(2)}$ the conditional expectation

$$
\bar{\sigma}_{1}^{(2)}: a_{1} \otimes b_{1} \in \mathcal{B}\left(\mathcal{H}_{1}\right) \otimes \mathcal{B}\left(\mathcal{K}_{1}\right) \rightarrow a_{1} \sigma_{1}\left(b_{1}\right) \in \mathcal{B}\left(\mathcal{H}_{1}\right) \cong \mathcal{B}\left(\mathcal{H}_{1}\right) \otimes 1_{\mathcal{K}_{1}}
$$

Then the lifting and the channel describing quantum communication processes are defined by

$$
\begin{gathered}
\mathcal{E}^{*}=\gamma^{*} \circ \bar{\sigma}_{1}^{(2) *} \\
\Lambda^{*} \rho=\alpha^{*} \circ \mathcal{E}^{*}(\rho)=\operatorname{tr}_{\mathcal{K}_{2}} \gamma^{*}\left(\rho \otimes \sigma_{1}\right) ; \rho \in \mathcal{S}\left(\mathcal{B}\left(\mathcal{H}_{1}\right)\right)
\end{gathered}
$$


where $\rho$ and $\sigma_{1}$ correspond to an input state and a noise state, respectively (c.f. [21]).

Moreover the following remark, extending an unpublished result of A.Frigerio, shows that the above model of the quantum communication process is universal among the transition expectations, provided one chooses the space of the representation large enough.

Theorem 1.4: Let $\mathcal{B}=\mathcal{B}(\mathcal{H})$ for a separable, infinite dimensional, Hilbert space $\mathcal{H}$ and let $\mathcal{E}: \mathcal{B} \otimes \mathcal{B} \longrightarrow \mathcal{B}$ be a normal transition expectation. Then there exist a normal state $\varphi$ on the $W^{*}$-algebra $(\otimes \mathcal{B})^{3} \otimes \mathbf{M}_{2}=: \mathcal{C}\left(\mathbf{M}_{2}\right.$ is the algebra of $2 \times 2$ complex matrices), and a unitary element $U$ of

$$
\mathcal{A}:=\mathcal{C} \otimes \mathcal{B} \cong(\otimes \mathcal{B})^{4} \otimes \mathbf{M}_{2}
$$

such that , denoting $\pi: \mathcal{B} \otimes \mathcal{B} \rightarrow \mathcal{A}$ the normal representation (amplification)

$$
\pi(x)=\left(1_{\mathcal{B}^{2}} \otimes x\right) 0
$$

Proof: From Kraus' Lemma [18] we know that, since $\mathcal{E}$ is normal, there exist a coutable (since $\mathcal{H}$ is separable family) $a_{i} \in \mathcal{B} \otimes \mathcal{B}(i \in N)$ such that , identifying $\mathcal{B}$ with $\mathcal{B} \otimes 1$ one has

$$
\mathcal{E}(x)=\sum_{i \in N} a_{i}^{*} x a_{i} \quad x \in \mathcal{B} \otimes \mathcal{B}
$$

If $\mathcal{H}$ is infinite dimensional in $\mathcal{B} \otimes \mathcal{B}$ there exist isometries $u_{i}(i \in N)$ such that for each $i, j u_{i}^{*} u_{j}=\delta_{i j}$. Thus, defining

$$
v:=\sum_{i=1}^{d} u_{i} \otimes a_{i} \in(\otimes \mathcal{B})^{4}
$$

one finds

$$
v^{*}\left(1_{\mathcal{B}^{2}} \otimes x\right) v=1_{\mathcal{B}^{2}} \otimes \mathcal{E}(x) \quad x \in \mathcal{B} \otimes \mathcal{B}
$$

In particular, since $v^{*} v=1_{\mathcal{B}^{4}}, v$ is a partial isometry with initial projection the identity. Denote $e=v v^{*}$ its final projection and define the unitary operator

$$
U=(v) 1-e
$$

\section{CONVEX COMBINATIONS OF PRODUCT STATES}

One of the main differences between classical and quantum probability is that, while all the measures on a product space are in the closed convex hull (for the weak topology) of product measures, it is not true that all the states on the tensor product $\mathcal{A}_{1} \otimes \mathcal{A}_{2}$ of two general $C^{*}$-algebras are limits (in some topology) of convex combinations of product states.

In particular, the image under a general lifting $\mathcal{E}^{*}$ of a state $\varphi$ will usually not be a convex combination of product states. 
However the class of liftings with this porperty is particulary interesting because we expect that in this class some features of quantum probability will mix with some features of classical probability. This class is defined as follows:

Definition 2.1: Let $\mathcal{A}_{1}$ and $\mathcal{A}_{2}$ be $W^{*}$-algebras. A lifting $\mathcal{E}^{*}: \mathcal{S}\left(\mathcal{A}_{1}\right) \rightarrow \mathcal{S}\left(\mathcal{A}_{1} \otimes\right.$ $\mathcal{A}_{2}$ ) will be called of convex product type, or shortly a convex product lifting, if any state $\omega \in \mathcal{S}\left(\mathcal{A}_{1}\right)$ is mapped by $\mathcal{E}^{*}$ into a convex combination of product states on $\mathcal{A}_{1} \otimes \mathcal{A}_{2}$. If this property holds only for any state $\omega$ in a subset $\mathcal{F} \subseteq \mathcal{S}\left(\mathcal{A}_{1}\right)$ then $\mathcal{E}^{*}$ is called a convex product lifting with respect to the family $\mathcal{F}$.

For any von Neumann algebra $\mathcal{A}$, the set $\mathcal{S}(\mathcal{A})$ of all its states has a natural structure of measurable space with its Borel $\sigma$-algebra. In the following any probability measure on $\mathcal{S}(\mathcal{A})$ will be meant with respect to this $\sigma$-algebra.

Definition 2.2: A convex decomposition of $\varphi \in \mathcal{S}(\mathcal{A})$ is a probability measure $\mu$ on $\mathcal{S}(\mathcal{A})$ satisfying

$$
\varphi=\int_{\mathcal{S}(\mathcal{A})} \omega d \mu(\omega)
$$

If $\mu$ is pseudosupported, in the sense of [8], in the set of extremal states of $\mathcal{S}(\mathcal{A})$, we speak of an extremal convex decomposition of $\varphi$.

Proposition 2.1: To every lifting of convex product type $\mathcal{E}^{*}: \mathcal{S}\left(\mathcal{A}_{1}\right) \rightarrow \mathcal{S}\left(\mathcal{A}_{1} \otimes \mathcal{A}_{2}\right)$, one can associate a pair

$$
\left\{p_{\rho}\left(d \omega_{1}\right), p_{\rho}\left(d \omega_{2} \mid \omega_{1}\right)\right\}
$$

with the following properties:

(i) $p_{\rho}\left(d \omega_{1}\right)$ is a probability measure on $\mathcal{S}\left(\mathcal{A}_{1}\right)$

(ii) $p_{\rho}\left(d \omega_{2} \mid \omega_{1}\right)$ is a Markovian kernel from $\mathcal{S}\left(\mathcal{A}_{1}\right)$ to $\mathcal{S}\left(\mathcal{A}_{2}\right)$. Conversely every pair (2.2) satisfying (i) and (ii) above determines, via (2.4) and (2.5), a unique convex product lifting.

Proof: For $\mathcal{E}^{*}$ as in Definition 2.1, let us fix a state $\rho_{1} \in \mathcal{S}\left(\mathcal{A}_{1}\right)$ and also a decomposition of $\mathcal{E}^{*} \rho_{1}$ as a convex combination of product states

$$
\mathcal{E}^{*} \rho_{1}=\int_{\mathcal{S}\left(\mathcal{A}_{1}\right) \times \mathcal{S}\left(\mathcal{A}_{2}\right)} \omega_{1} \otimes \omega_{2} d p\left(\omega_{1}, \omega_{2} \mid \rho_{1}\right)
$$

Denoting $p_{\rho_{1}}\left(d \omega_{2} \mid \omega_{1}\right)$ the conditional probability of $p\left(\cdot \mid \rho_{1}\right)$ on the $\sigma$-algebra of the first factor and $d p_{\rho_{1}}\left(\omega_{1}\right)$ the marginal of $p\left(\cdot \mid \rho_{1}\right)$ on the first factor, we obtain

$$
\begin{gathered}
\mathcal{E}^{*} \rho_{1}=\int_{\mathcal{S}\left(\mathcal{A}_{1}\right)} \int_{\mathcal{S}\left(\mathcal{A}_{2}\right)} \omega_{1} \otimes \omega_{2} d p_{\rho_{1}}\left(\omega_{1}\right) p_{\rho_{1}}\left(d \omega_{2} \mid \omega_{1}\right)=\int_{\mathcal{S}\left(\mathcal{A}_{1}\right)} \omega_{1} \otimes \Lambda_{\rho}^{*} \omega_{1} d p_{\rho_{1}}\left(\omega_{1}\right) \\
\Lambda_{\rho_{1}}^{*} \omega_{1}:=\int_{\mathcal{S}\left(\mathcal{A}_{2}\right)} \omega_{2} p_{\rho_{1}}\left(d \omega_{2} \mid \omega_{1}\right)
\end{gathered}
$$


Thus any lifting $\mathcal{E}^{*}: \mathcal{S}\left(\mathcal{A}_{1}\right) \rightarrow \mathcal{S}\left(\mathcal{A}_{1} \otimes \mathcal{A}_{2}\right)$, of convex product type, has the form (2.4), where $p_{\rho_{1}}$ is a probability measure on $\mathcal{S}\left(\mathcal{A}_{1}\right)$ and the map $\Lambda_{\rho_{1}}^{*}: \mathcal{S}\left(\mathcal{A}_{1}\right) \rightarrow \mathcal{S}\left(\mathcal{A}_{2}\right)$, is given by (2.5). Notice that $\Lambda_{\rho_{1}}^{*}$ is a channel in the sense of Section 1 and it is usually nonlinear both in $\omega_{1}$ and $\rho_{1}$.

Conversely, given $p_{\rho_{1}}$ and $\Lambda_{\rho_{1}}^{*}$ as above, if we define $\mathcal{E}^{*}$ by (2.3), then clearly $\mathcal{E}^{*}$ is a convex product lifting from $\mathcal{S}\left(\mathcal{A}_{1}\right)$ to $\mathcal{S}\left(\mathcal{A}_{1} \otimes \mathcal{A}_{2}\right)$. Finally it is clear that the map

$$
\left(\omega_{1}, S_{2}\right) \in \mathcal{S}\left(\mathcal{A}_{1}\right) \times \operatorname{Borel}\left(\mathcal{S}\left(\mathcal{A}_{2}\right)\right) \rightarrow p_{\rho_{1}}\left(\mathcal{S}_{2} \mid \omega_{1}\right) \in[0,1]
$$

is a classical Markovian kernel on the Borel space $\mathcal{S}\left(\mathcal{A}_{1}\right) \times \mathcal{S}\left(\mathcal{A}_{2}\right)$.

Remark: If in (2.3) one conditions on the $\sigma$-algebra of the second factor rather than on the first one, the resulting lifting is

$$
\mathcal{E}^{*} \rho_{1}=\int_{\mathcal{S}\left(\mathcal{A}_{1}\right)} \int_{\mathcal{S}\left(\mathcal{A}_{2}\right)} \omega_{1} \otimes \omega_{2} d q_{\rho_{1}}\left(\omega_{2}\right) d q_{\rho_{1}}\left(d \omega_{1} \mid \omega_{2}\right)
$$

where $d q_{\rho_{1}}\left(\omega_{2}\right)$ is a probability measure on $\mathcal{S}\left(\mathcal{A}_{2}\right)$ and $d q_{\rho_{1}}\left(d \omega_{1} \mid \omega_{2}\right)$ a Markovian kernel from $\mathcal{S}\left(\mathcal{A}_{2}\right)$ to $\mathcal{S}\left(\mathcal{A}_{1}\right)$.

Let us now consider the relation between the liftings of convex product type and Markov chains.

The dual of a linear lifting is a transition expectation, therefore to any linear lifting one can associate a quantum Markov chain [2] in a standard way.

If the lifting is of convex product type, then we can take advantage of this special structure to extend the construction of quantum Markov chains to the case of a not necessarily linear lifting $\mathcal{E}^{*}$. In what follows we describe this procedure.

If $\mathcal{E}^{*}: \mathcal{S}\left(\mathcal{A}_{2}\right) \rightarrow \mathcal{S}\left(\mathcal{A}_{1} \otimes \mathcal{A}_{2}\right)$ is a lifting of convex product type, then it has the form:

$$
\mathcal{E}^{*} \rho_{2}=\int_{\mathcal{S}\left(\mathcal{A}_{1}\right)} \int_{\mathcal{S}\left(\mathcal{A}_{2}\right)} \omega^{1} \otimes \omega^{2} p\left(d \omega^{1}, d \omega^{2} \mid \rho_{2}\right)
$$

Notice that $p\left(d \omega^{1}, d \omega^{2} \mid \rho_{2}\right)$ can be considered as a Markovian Kernel on the space

$$
\mathcal{S}_{12}:=\mathcal{S}\left(\mathcal{A}_{1}\right) \times \mathcal{S}\left(\mathcal{A}_{2}\right)
$$

which is constant on the first conditioning, i.e.,

$$
p\left(d \omega^{1}, d \omega^{2} \mid \rho_{1}, \rho_{2}\right)=p\left(d \omega^{1}, d \omega^{2} \mid \rho_{2}\right) \quad ; \quad \omega_{1}, \rho_{1}, \in \mathcal{S}\left(\mathcal{A}_{1}\right), \omega_{2}, \rho_{2} \in \mathcal{S}\left(\mathcal{A}_{2}\right)
$$

Clearly (2.6) is a state on $\mathcal{A}_{1} \otimes \mathcal{A}_{2}$. If we apply $\mathcal{E}^{*}$ to $\omega^{2}$ in (2.6), we obtain the following state on $\left(\mathcal{A}_{1} \otimes \mathcal{A}_{2}\right) \otimes \mathcal{A}_{2}$ :

$$
\int_{\mathcal{S}_{12}} p\left(d \omega_{1}^{1}, d \omega_{1}^{2}, \mid \rho_{2}\right) \omega_{1}^{1} \otimes \mathcal{E}^{*} \omega_{1}^{2}=
$$




$$
=\int_{\mathcal{S}_{12}} \int_{\mathcal{S}_{12}} p\left(d \omega_{1}^{1}, d \omega_{1}^{2} \mid \rho_{2}\right) p\left(d \omega_{2}^{1}, d \omega_{2}^{2} \mid \omega_{1}^{2}\right) \omega_{1}^{1} \otimes \omega_{2}^{1} \otimes \omega_{2}^{2}
$$

where

$$
\omega_{i}^{1} \in \mathcal{S}\left(\mathcal{A}_{1}\right) \text { and } \omega_{i}^{2} \in \mathcal{S}\left(\mathcal{A}_{2}\right) .
$$

Applying again $\mathcal{E}^{*}$ to $\omega_{2}^{2}$ we find

$$
\int_{\mathcal{S}_{12}} \int_{\mathcal{S}_{12}} \int_{\mathcal{S}_{12}} p\left(d \omega_{1}^{1}, d \omega_{1}^{2} \mid \rho_{2}\right) p\left(d \omega_{2}^{1}, d \omega_{2}^{2} \mid \omega_{1}^{2}\right) p\left(d \omega_{3}^{1}, d \omega_{3}^{2} \mid \omega_{2}^{2}\right) \omega_{1}^{1} \otimes \omega_{2}^{1} \otimes \omega_{3}^{1} \otimes \omega_{3}^{2}
$$

At the $n$-th iteration we obtain the state $\mathcal{E}_{n]}^{*} \rho_{2}$ on $\left(\otimes \mathcal{A}_{1}\right)^{n} \otimes \mathcal{A}_{2}$, defined by:

$$
\mathcal{E}_{n]}^{*} \rho_{2}:=\int_{\mathcal{S}_{12}^{n}}\left(\otimes_{i=1}^{n} \omega_{i}^{1}\right) \otimes \omega_{n}^{2} \cdot \Pi_{i=2}^{n} p\left(d \omega_{i}^{1}, d \omega_{i}^{2} \mid \omega_{i-1}^{2}\right) p\left(d \omega_{1}^{1}, d \omega_{1}^{2}, \mid \rho_{2}\right)
$$

This suggests to introduce the classical Markov process

$$
\xi_{n}:=\left(\xi_{n}^{1}, \xi_{n}^{2}\right):(\Omega, \mathcal{F}, P) \rightarrow \mathcal{S}\left(\mathcal{A}_{1}\right) \times \mathcal{S}\left(\mathcal{A}_{2}\right)=S_{12}
$$

with the transition function given by $(2.7)$ and initial distribution $p\left(\cdot \mid \rho_{2}\right)$. This transition probability has a nice interpretation in terms of signal + noise : if system1 represents the noise and system 2 the signal, then condition (2.7) means that the joint distribution at time $(n+1)$ depends on the signal at time $n$, but not on the noise at time $n$ : a natural assumption if we think of the noises at defferent times, as generated by independent causes. Now let $\mathcal{A}:=\otimes_{\mathbf{N}} \mathcal{A}_{1}$. The identification

$$
a_{1} \otimes \ldots \otimes a_{n} \cong a_{1} \otimes a_{2} \otimes \ldots \otimes a_{n} \otimes 1 \otimes 1 \otimes \cdots
$$

induces a natural identification of $\left(\otimes \mathcal{A}_{1}\right)^{n}$ with a sub-algebra $\mathcal{A}_{[2, n]}$ of $\mathcal{A}=\otimes_{\mathbf{N}} \mathcal{A}_{1}$ (the product of the first $n$-factors).

In particular, if $\rho_{2} \in \mathcal{S}\left(\mathcal{A}_{2}\right)$ is a state on $\mathcal{A}_{2}$, the restriction of $\mathcal{E}_{n]}^{*} \rho_{2}$ on $\left(\otimes \mathcal{A}_{1}\right)^{n}$ is a state on $\left(\otimes^{n} \mathcal{A}_{1}\right)$ and, with the above identification, we can consider it a state $\rho_{[1, n]}$ on $\mathcal{A}$. Following from all above, in particular (2.8), we obtain

Proposition 2.2: For any $\rho_{2} \in \mathcal{S}\left(\mathcal{A}_{2}\right)$ the limit

$$
\lim _{n \rightarrow \infty} \rho_{[1, n]}=: \varphi
$$

exists pointwise weakly on $\mathcal{A}$. Moreover, if $E_{\xi}$ denotes the mean with respect to the process $\left\{\xi_{n}\right\}$, defined by $(2.9)$, then one has

$$
\varphi=E_{\xi}\left(\otimes_{n \in \mathbf{N}} \xi_{n}^{1}\right)
$$

\section{CENTRALIZER LIFTINGS}


In this Section we introduce an interesting class of nonlinear liftings generalizing the construction of [22]. It is shown that the Cecchini-Petz notion of state extension [11], introduced after [22] and for totally independent reasons, is a generalization of our construction hence, a fortiori, of the one in [22].

Recall that a linear map $E$ from a $\mathrm{C}^{*}$-algebra $\mathcal{A}$ to a $\mathrm{C}^{*}$-algebra $\mathcal{B}$ is called anticompletely positive if the map $\bar{E}: \mathcal{A} \rightarrow \mathcal{B}$, defined by

$$
\bar{E}(a):=E\left(a^{*}\right) \quad ; \quad a \in \mathcal{A}
$$

is completely positive antilinear, i.e., for any natural integer $n$, any $a_{1}, \ldots, a_{n} \in \mathcal{A}$ and any $b_{1}, \ldots, b_{n} \in \mathcal{B}$, one has

$$
\sum_{j k} b_{j}^{*} E\left(a_{k}^{*} a_{j}\right) b_{k}=\sum_{j k} b_{j}^{*} \bar{E}\left(a_{j}^{*} a_{k}\right) b_{k} \geq 0
$$

Proposition 3.1: Let $\mathcal{A}_{1}, \mathcal{A}_{2}$ be $\mathrm{W}^{*}$-algebras, let $\mathcal{A}_{1} \otimes^{\left({ }^{o}\right)} \mathcal{A}_{2}$ denote their algebraic tensor product. For $\rho \in \mathcal{S}\left(\mathcal{A}_{1}\right)$ let $\mathcal{A}_{1}^{\rho}$ denote the centralizer of $\rho$ and let $E: \mathcal{A}_{2} \rightarrow \mathcal{A}_{1}^{\rho}$ be any anticompletely positive identity preserving linear map. Then there exists a unique state $\varphi_{\rho}$ on $\mathcal{A}_{1} \otimes^{(o)} \mathcal{A}_{2}$ such that

$$
\varphi_{\rho}\left(a_{1} \otimes a_{2}\right):=\rho\left(a_{1} E\left(a_{2}\right)\right) \quad ; \quad a_{1} \in \mathcal{A}_{1}, a_{2} \in \mathcal{A}_{2}
$$

Proof: Let $n$ be a natural integer and let $b_{1}, \ldots, b_{n} \in \mathcal{A}_{1}$ and $a_{1}, \ldots, a_{n} \in \mathcal{A}_{2}$. By assumption the $\mathcal{A}_{1}^{\rho}$-valued $n \times n$ matrix $B=\left(B_{k j}\right)$ defined by

$$
B_{k j}:=E\left(a_{j}^{*} a_{k}\right)=\bar{E}\left(a_{k}^{*} a_{j}\right)
$$

is of positive type, hence it has the form $B=M^{*} M$ for some $\mathcal{A}_{1}^{\rho}$-valued $n \times n$ matrix $M=\left(M_{k j}\right)$. One has therefore

$$
\begin{gathered}
\varphi_{\rho}\left(\left|\sum_{j} a_{j} \otimes b_{j}\right|^{2}\right)=\sum_{j k} \rho\left(b_{j}^{*} b_{k} E\left(a_{j}^{*} a_{k}\right)\right)= \\
=\sum_{j k h} \rho\left(b_{j}^{*} b_{k} M_{h k}^{*} M_{h j}\right)= \\
=\sum_{j k h} \rho\left(M_{h j} b_{j}^{*} b_{k} M_{h k}^{*}\right)= \\
=\sum_{h} \rho\left(\left[\sum_{j} b_{j} M_{h j}^{*}\right]^{*} \cdot\left[\sum_{k} b_{k} M_{h k}^{*}\right]\right) \geq 0
\end{gathered}
$$




\section{Remark: Clearly}

$$
\left|\varphi_{\rho}\left(a_{1} \otimes a_{2}\right)\right| \leq\left\|a_{1}\right\| \cdot\left\|a_{2}\right\| \quad ; \quad a_{1} \in \mathcal{A}_{1}, a_{2} \in \mathcal{A}_{2}
$$

hence $\varphi_{\rho}$ is continuous for the greatest cross norm on $\mathcal{A}_{1} \otimes^{(o)} \mathcal{A}_{2}$. Cecchini and Petz [12] have proved that it is also continuous for the smallest $\mathrm{C}^{*}$-norm [28]. (This is clear if the centralizer of $\rho$, i.e., $\mathcal{A}_{1}^{\rho}$, is abelian because in that case all the $\mathrm{C}^{*}$-norms on $\mathcal{A}_{1} \otimes^{\left({ }^{o}\right)} \mathcal{A}_{2}$ coincide with the minimal $\mathrm{C}^{*}$-norm ([28], Proposition 1.22.5)). Moreover it is easy to check that the operator $E$, defined by (3.1) is an example of Cecchini's $\lambda$-operator [9]. In this case in fact the Tomita involution $J_{1}$ acts as the identity on the cyclic space of $\mathcal{A}_{1}^{\rho}$, the centralizer of $\mathcal{A}_{1}$, therefore the identity (3.1) is precisely the defining relation of the $\lambda$-operator.

If $\mathcal{A}_{1}^{\rho}$ is a discrete abelian algebra generated by a partition $\left(e_{j}\right)$ of the identity, then any positive map $E$, from $\mathcal{A}_{2}$ to $\mathcal{A}_{1}^{\rho}$ is also completely and anti-completely positive and it has the form

$$
E\left(a_{2}\right)=\sum_{j} \varphi_{j}\left(a_{2}\right) e_{j} \quad ; \quad a_{2} \in \mathcal{A}_{2}
$$

with $\varphi_{j} \in \mathcal{S}\left(\mathcal{A}_{2}\right)$. In this case it is immediate to verify that

$$
\varphi_{\rho}=\sum_{j} \rho_{j} \otimes \varphi_{j}
$$

where $\varphi_{j}$ is given by $(3.2)$ and

$$
\rho_{j}:=\rho\left(e_{j}(\cdot) e_{j}\right)
$$

In general, whenever the state $\varphi_{\rho}$, defined by (3.3), is continuous, the map $\rho \mapsto \varphi_{\rho}$ defines a lifting $\mathcal{E}^{*}$ in the sense of Definition 1.1. This lifting is in general nonlinear since the map $E$ in (3.1) may depend on $\rho$.

For example, if $\mathcal{A}_{1}$ is the algebra of all operators on some Hilbert space and $\rho$ has the form $\rho=\operatorname{tr}(w \cdot)$ for some density matrix $w$ with spectral decomposition given by

$$
w=\sum_{j} p_{j} e_{j}
$$

then the centralizer $\mathcal{A}_{1}^{\rho}$ is the closed linear span of the $\left(e_{j}\right)$ and if the $\varphi_{j}$ are chosen to be of the form

$$
\varphi_{j}=\Lambda^{*}\left(\frac{\rho_{j}}{\rho_{j}(1)}\right)
$$

for some channel $\Lambda^{*}: \mathcal{S}\left(\mathcal{A}_{1}\right) \rightarrow \mathcal{S}\left(\mathcal{A}_{2}\right)$, then equation (3.3) becomes of the same form as (1.17) giving an example of nonlinear compound lifting.

\section{ERROR PROBABILITY FOR OPTICAL COMMUNICATION}


An optical communication process studied by several authors (cf.[21] for a mathematical analysis), the so-called attenuation process, can be described by the isometric lifting described in Example 1.a. This description is simpler than the previous ones and allows quicker computations. This statement is illustrated with the computation of several error probabilities related to this model.

Before introducing these computations, we briefly review some basic facts about the notions of quantum coding and of error probability in quantum control communication processes along the lines of [24].

Suppose that, by some procedure, we encode an information representing it by a sequence of letters $c^{(1)}, \ldots, c^{(n)}, \ldots$, where $c^{(k)}$ is an element in a set $\mathcal{C}$ of symbols called the alphabet.

A quantum code is a map which associates to each symbol (or sequence of symbols) in $\mathcal{C}$ a quantum state, representing an optical signal. Sometimes one uses a state as two codes: one for input and one for output.

In the sequel we shall only consider a two symbols alphabet:

$$
\mathcal{C}=\{0,1\}
$$

One example of quantum code $\Xi=\left\{\xi_{0}, \xi_{1}\right\}$ where $\xi_{i}$ is the quantum state corresponding to the symbol $c_{i} \in \mathcal{C}$, is obtained by choosing $\xi_{0}$ as the vacuum state and $\xi_{1}$ another state such as a coherent or a squeezed state of a one-mode field.

Two states (quantum codes) $\xi_{0}^{(1)}$ and $\xi_{1}^{(1)}$ in the input system are transmitted to the output system through a channel $\Lambda^{*}$. We here assume a Z-type signal transmission, namely that the input signal "0", represented by the state $\xi_{0}^{(1)}$, is error free in the sense that it goes always to the output signal "0" represented by $\xi_{0}^{(2)}$, while the input signal "1", represented by the state $\xi_{1}^{(1)}$, is not error free in the sense that its output can give rise to both states $\xi_{0}^{(2)}$ or $\xi_{1}^{(2)}$ with different probabilities.

The error probability $q_{e}$ is then the probability that the input signal " 1 " is recognized as the output signal " 0 ", so that it is given by

$$
q_{e}=\operatorname{tr} \Lambda^{*}\left(\xi_{1}^{(1)}\right) \xi_{0}^{(2)}
$$

In the case of quantum attenuation process, this error probability is written by using the attenuation operator $V$ given in Example (1.a) with the construction (Example (4)) of quantum lifting:

$$
q_{e}=\operatorname{tr}_{\mathcal{H}_{2}} \operatorname{tr}_{\mathcal{K}_{2}}\left(V \xi_{1}^{(1)} V^{*}\right) \xi_{0}^{(2)}
$$

There are two main ways, called pulse modulation, to code the symbols of the alphabet $\mathcal{C}$. We briefly explain them for completeness. A pulse is an optical signal, represented by a non vacuum state of the EM field; its energy is here called the height of the pulse. To a single symbol of the alphabet $\mathcal{C}$ one associates one or more pulses. Time is discretized and each time interval between $t_{k}$ and $t_{k+1}$ has length $\tau$. Each time interval corresponds to a single symbol of the alphabet. 
(1) PCM (Pulse Code Modulation) : To the $k$-th symbol $a_{k}$ of the input sequence, one associates $N$ pulses starting at a time $t_{k}$. The ordered set of these pulses is denoted $x_{k}$. For instance, for the alphabet $\left\{a_{0}, a_{1}\right\}$, for $N=5$ and choosing the elementary pulses to be the vacuum (i.e., no pulse) denoted 0 , and another fixed pulse, e.g., a coherent state, denoted 1 , the code $x_{k}$ corresponding to $a_{k}$ is determined by $x_{0}=(1,0,1,0,0), x_{1}=(0,1,1,0,1)$ and so on. For this modulation, we need $N$ slots (sites) in one time interval (e.g., between $t_{k}$ and $t_{k+1}$ ) to fully represent all $\mathrm{M}$ signals; $2^{N-1}<M \leq 2^{N}$.

(2) PPM (Pulse Position Modulation) : In this case there is only one nonvacuum pulse in each time interval of length $\tau$. The code $x_{k}$ expressing a signal $a_{k}$ is determined by the position of the non vacuum pulse, so that we need $M$ slots (sites) in each time interval in order to express $M$ signals; For instance in the same notations as above, $x_{0}=(0,0,0,1,0), x_{2}=(0,1,0,0,0)$.

Given (4.2), the error probability of PCM with the $t_{0}$-tuple error correcting the following (4.3) and (4.4), respectively:

$$
\begin{aligned}
P_{e}^{P C M} & =\sum_{j=t_{0}+1}^{\nu}{ }_{\nu} C_{j} q_{e}^{j}\left(1-q_{e}\right)^{\nu-j}, \\
P_{e}^{P P M} & =q_{e}
\end{aligned}
$$

where ${ }_{\nu} C_{j}=\nu ! /\{(\nu-\mathrm{j}) ! \mathrm{j} !\}$.

The most general case for the computation of $q_{e}$ is one that both $\xi_{1}^{(1)}$ and $\xi_{0}^{(2)}$ are squeezed states, but in usual optical communication it is often enough to take a coherent or squeezed state as $\xi_{1}^{(1)}$ and the vacuum state as $\xi_{0}^{(2)}$. Hence we first calculate the error probability $q_{e}$ for the latter two cases and compare them with the results previously obtained in [24]. Secondly we show the computation for the most general case, $\xi_{1}^{(1)}$ and $\xi_{0}^{(2)}$ squeezed, for a mathematical interest and generality, although this somehow does not fit to the assumption of our $\mathrm{Z}$ type transmission.

(I) Case of $\xi_{1}^{(1)}=|\theta><\theta|=$ coherent state and $=|0><0|$ : The error probability (4.2) becomes

$$
\begin{aligned}
q_{e} & =\operatorname{tr}_{\mathcal{H}_{2}}\left(\operatorname{tr}_{\mathcal{K}_{2}} V^{*}|\theta><\theta| V\right)|0><0| \\
& =\operatorname{tr}_{\mathcal{H}_{2}}\left(\operatorname{tr}_{\mathcal{K}_{2}}|\alpha \theta><\alpha \theta| \otimes|\beta \theta><\beta \theta|\right)|0><0| \\
& =\operatorname{tr}_{\mathcal{H}_{2}}|\alpha \theta><\alpha \theta||0><0| \\
& =|<0, \alpha \theta>|^{2} \\
& =\exp \left(-|\alpha \theta|^{2}\right),
\end{aligned}
$$

which is equal to the usual result (cf. [16], [19]), but our new derivation is much simpler than old one. 
(II) Case of $\xi_{1}^{(1)}=$ squeezed state and $\xi_{0}^{(2)}=|0><0|$ : A squeezed state can be expressed by a unitary operator $U(z)(z \in \mathbf{C})$ given in Appendix such that

$$
\xi_{1}^{(1)}=U(z)|\gamma><\gamma| U(z)^{*}
$$

where $|\gamma\rangle$ is a certain coherent state. Therefore the error probability $q_{e}$ is

$$
\begin{aligned}
q_{e} & =\operatorname{tr}_{\mathcal{H}_{2}}\left(\operatorname{tr}_{\mathcal{K}_{2}} V U(z)|\gamma><\gamma| U(z)^{*} V^{*}\right)|0><0| \\
& =\operatorname{tr} V^{*}(|0><0| \otimes I) V U(z)|\gamma><\gamma| U(z)^{*} \\
& =<U(z) \gamma, V^{*}(|0><0| \otimes I) V U(z) \gamma>
\end{aligned}
$$

To carry this calculation, we have to know the effect of $V^{*}$ on $\mathcal{H}_{2} \otimes \mathcal{K}_{2}$.

$$
\begin{aligned}
<\gamma, & \left(V^{*}\left|\gamma^{\prime}>\otimes\right| \gamma^{\prime \prime}>\right)>=<V \gamma,\left(\left|\gamma^{\prime}>\otimes\right| \gamma^{\prime \prime}>\right)> \\
= & <(<\alpha \gamma|\otimes<\beta \gamma|),\left(\left|\gamma^{\prime}>\otimes\right| \gamma^{\prime \prime}>\right)> \\
= & <\alpha \gamma, \gamma^{\prime}><\beta \gamma, \gamma^{\prime \prime}> \\
= & \exp \left\{\frac{1}{2}\left(-|\alpha \gamma|^{2}-\left|\gamma^{\prime}\right|^{2}+2 \bar{\alpha} \bar{\gamma} \gamma^{\prime}\right)\right\} \exp \left\{\frac{1}{2}\left(-\left|\gamma^{\prime \prime}\right|^{2}-|\beta \gamma|^{2}+2 \bar{\beta} \bar{\gamma} \gamma^{\prime \prime}\right)\right\} \\
= & \exp \left\{-\frac{1}{2}\left(\left|\beta \gamma^{\prime}\right|^{2}+\left|\alpha \gamma^{\prime \prime}\right|^{2}\right)\right\}<\gamma, \bar{\alpha} \gamma^{\prime}+\bar{\beta} \gamma^{\prime \prime}>\exp \left(\operatorname{Re}\left(\bar{\alpha} \beta \gamma^{\prime} \bar{\gamma} "\right)\right),
\end{aligned}
$$

which implies

$$
\begin{aligned}
V^{*}\left|\gamma^{\prime}>\otimes\right| \gamma^{\prime \prime}> & =\exp \left\{-\frac{1}{2}\left(\left|\beta \gamma^{\prime}\right|^{2}+\left|\alpha \gamma^{\prime \prime}\right|^{2}\right)\right\} \\
& \times \exp \left(\operatorname{Re}\left(\bar{\alpha} \beta \gamma^{\prime} \gamma^{\prime \prime}\right)\right) \mid \bar{\alpha} \gamma^{\prime}+\bar{\beta} \gamma^{\prime \prime}>
\end{aligned}
$$

Therefore $q_{e}$ is

$$
\begin{aligned}
q_{e} & =\frac{1}{\pi} \int d^{2} w<U(z) \gamma, V^{*}(|0><0| \otimes I) V w><w, U(z) \gamma> \\
& =\frac{1}{\pi} \int d^{2} w \exp \left\{\frac{1}{2}\left(|\alpha|^{2}|\beta|^{2}|w|^{2}\right)\right\} \\
& \times<U(z) \gamma,|\beta|^{2} w><0, \alpha w><w, U(z) \gamma>
\end{aligned}
$$

This can be computed by the expression (A.27) given in Appendix and a Gaussian type integration:

$$
\begin{aligned}
<w, U(z) \gamma>= & <\exp \left(-\frac{i}{2} \varphi\right) w, U(r) \exp \left(-\frac{i}{2} \varphi\right) \gamma> \\
& =\exp \left\{-\frac{1}{2}\left(|w|^{2}+|\gamma|^{2}\right)\right\}(\cosh r)^{-1 / 2} \\
& \times \exp \left\{\bar{w} \theta(\cosh r)^{-1}+\tanh r\left\{\frac{1}{2}\left(\exp (-i \varphi) \theta^{2}-\exp (i \varphi) \bar{w}^{2}\right)\right\}\right\},
\end{aligned}
$$




$$
\frac{1}{\pi} \int d^{2} w \exp \left\{-|w|^{2}+a w+b \bar{w}+c w^{2}+d \bar{w}^{2}\right\}=\frac{1}{\sqrt{1-4 c d}} \exp \left\{\frac{a^{2} d+a b+b^{2} c}{1-4 c d}\right\} .
$$

The result is

$$
\begin{aligned}
q_{e}= & \frac{(\cosh r)^{-1} \exp \left\{\frac{1}{2}\left(\gamma^{2}+\bar{\gamma}^{2}\right)(\tanh r)-|\gamma|^{2}\right\}}{\sqrt{1-\left(1-|\alpha|^{2}\right)^{2}(\tanh r)^{2}}} \\
& \times \exp \left(\frac{-\frac{1}{2}\left(1-|\alpha|^{2}\right)^{2}(\cosh r)^{-2}(\tanh r)\left(\gamma^{2}+\bar{\gamma}^{2}\right)+\left(1-|\alpha|^{2}\right)|\gamma|^{2}(\cosh r)^{-2}}{1-\left(1-|\alpha|^{2}\right)^{2}(\tanh r)^{2}}\right) \\
& =\frac{1}{\sqrt{(\cosh r)^{2}-\left(1-|\alpha|^{2}\right)^{2}(\sinh r)^{2}}} \exp \left\{\left(\frac{1-|\alpha|^{2}}{(\cosh r)^{2}-\left(1-|\alpha|^{2}\right)^{2}(\sinh r)^{2}}-1\right)|\gamma|^{2}\right. \\
& \left.+\left(1-\frac{\left(1-|\alpha|^{2}\right)^{2}}{(\cosh r)^{2}-\left(1-|\alpha|^{2}\right)^{2}(\sinh r)^{2}}\right)\left(\frac{1}{2}\left(\gamma^{2}+\bar{\gamma}^{2}\right)(\tanh r)\right)\right\}
\end{aligned}
$$

which is same as the result obtained in [24]:

$$
\begin{aligned}
q_{e} & =\sqrt{\tau} \exp \left[\{(1-\eta) \tau-1\}|\gamma|^{2}\right. \\
& \left.+\left[1-(1-\eta)^{2} \tau\right]\left\{\frac{\bar{\mu} \gamma^{2}}{2 \lambda}+\frac{\mu \bar{\gamma}^{2}}{2 \bar{\lambda}}\right\}\right]
\end{aligned}
$$

where $\eta=|\alpha|^{2}, \tau=\left\{|\lambda|^{2}-(1-\eta)^{2}|\mu|^{2}\right\}^{-1}$ with $\lambda=\exp (i \phi) \cosh r, \mu=\sinh r$.

(III) Case of $\xi_{1}^{(1)}=$ squeezed state $U(p)|\gamma><\gamma| U(p)^{*}$ and $\xi_{0}^{(2)}=$ squeezed state $U(q)|\sigma><\sigma| U(q)^{*}$ : By the similar way as the case (II),

$$
\begin{aligned}
q_{e} & =\operatorname{tr}_{\mathcal{H}_{2}}\left(\operatorname{tr}_{\mathcal{K}_{2}} V U(p)|\gamma><\gamma| U(p)^{*} V^{*}\right) U(q)|\sigma><\sigma| U(q)^{*} \\
& =<U(p) \gamma, V^{*}(|U(q) \sigma><U(q) \sigma| \otimes I) V U(p) \gamma> \\
& =\frac{1}{\pi^{2}} d^{2} w d^{2} z<U(p) \gamma, w><w, V^{*}(|U(q) \sigma><U(q) \sigma| \otimes I) V z><z, U(p) \gamma> \\
& =\frac{1}{\pi^{2}} d^{2} w d^{2} z<U(p) \gamma, w><\alpha w, U(q) \sigma><\beta w, \beta z> \\
& \times<U(q) \sigma, \alpha z><z, U(p) \gamma>
\end{aligned}
$$

Applying the above formula presented in the case II, we can compute this error probability $q_{e}$ as

$$
q_{e}=\frac{1}{\pi^{2}}<U(p) \gamma, w><\alpha w, U(q) \sigma><\beta w, \beta z>
$$

Let $\mathbf{C}$ denote the set of all complex numbers. A Fock representation of the Canonical Commutation Relations (CCR) over $\mathbf{C}$ is a triple

$$
\{\mathcal{H}, W, \Phi\}
$$


where $\mathcal{H}$ is a Hilbert space and $W: z \in \mathbf{C} \mapsto W(z) \in U_{n}(\mathcal{H})$ is a map from $\mathbf{C}$ to the unitary operators on $\mathcal{H}$ such that $W(0)=$ id and

$$
W(u) W(v)=\exp \{i \operatorname{Im} \bar{u} v\} W(u+v) ; u, v \in \mathbf{C}
$$

and $\Phi \in \mathcal{H}$ is a unit vector, called the Fock vacuum, satisfying

$$
<\Phi, W(z) \Phi>=\exp \left\{-12|z|^{2}\right\} ; \quad z \in \mathbf{C}
$$

It is moreover assumed that the weak closure of the complex vector space generated by the $\{W(z): z \in \mathbf{C}\}$ coincides with the algebra of all bounded operators on $\mathcal{H}$. This property is called irreducibility. Clearly any two Fock representations are canonically isomorphic. The Stone-von Neumann theorem asserts that if $\{W(z): z \in \mathbf{C}\}$ is any irreducible family of unitary operators on a Hilbert space $\mathcal{H}$ satisfying (A.1), then it is isomorphic to the Fock representation. In particular, for any such a family, there will exist a (necessarily unique) vector $\Phi$ satisfying (A.2), i.e., a Fock vacuum for this family. A corollary of the Stone-von Neumann theorem is the following: let $T: \mathbf{C} \rightarrow \mathbf{C}$ be any real linear transformation such that

$$
\operatorname{Im}(T u)^{-}(T v)=\operatorname{Im} \bar{u} v ; \forall u, v \in \mathbf{C}
$$

where "-" denotes the complex conjugate, and define

$$
W_{T}(z)=W(T z) ; z \in \mathbf{C}
$$

Then the set $\left\{W_{T}(z): z \in \mathbf{C}\right\}$ (because any $T$ satisfying (A.3) must be invertible), hence it is irreducible. Moreover, because of (A.3), it satisfies (A.1). Hence by the Stone-von Neumann theorem, there exists a vector $\Phi_{T} \in \mathcal{H}$ and a unitary operator $U_{T}: \mathcal{H} \rightarrow \mathcal{H}$, characterized by the property:

$$
U_{T} W(z) \Phi=W_{T}(z) \Phi_{T} ; z \in \mathbf{C}
$$

The vector $\Phi_{T}=U_{T} \Phi$ (i.e., the vacuum for the $W_{T}$ representation) is called a squeezed vector for the $\mathrm{W}$-representation. The most general operator $T$, satisfying (A.3), is given by the following

Proposition A.1: Let $V: \mathbf{C} \rightarrow \mathbf{R}^{2}$ be the isomorphism of real linear spaces characterized by

$$
V(1)=(1)
$$

Then a real $2 \times 2$ matrix $T$ induces on $\mathbf{C}$ a transformation satisfying (A.3) if and only if $\operatorname{det} T=1$.

Proof: A direct calculation: The identity (A.2) implies that for each $z \in \mathbf{C}$ the one parameter unitary group $\{W(t z)\}(t \in \mathbf{R})$ is strongly continuous, hence

$$
W(t z)=\exp \{i t B(z)\}
$$


for some self-adjoint operator $B(z)$. Moreover the map $z \in \mathbf{C} \mapsto B(z)$ is real linear. The operators

$$
12 B(1)=q ; 12 B(i)=p
$$

are called momentum and position operators, respectively. The condition (A.1) implies that

$$
[B(u), B(v)]=2 i \operatorname{Im} \bar{u} v
$$

so that, in particular

$$
[q, p]=i 2
$$

Finally, denoting

$$
a=p-i q ; a^{*}=p+i q
$$

one has

$$
\begin{gathered}
{\left[a, a^{*}\right]=1} \\
i B(z)=z a^{*}-\bar{z} a ; \quad z \in \mathbf{C}
\end{gathered}
$$

The vectors

$$
\mid \theta>=W(\theta) \Phi ; \theta \in \mathbf{C}
$$

are called coherent vectors. Now let $T: \mathbf{C} \rightarrow \mathbf{C}$ be a real linear map satisfying (A.3) and let $W_{T}, U_{T}, \Phi_{T}$ be characterized respectively by (A.4) and (A.5). Then one has, for $z \in \mathbf{C}$ :

$$
W_{T}(z)=\exp \left(i B_{T}(z)\right)=\exp \left(z a_{T}^{*}-\bar{z} a_{T}\right) .
$$

On the other hand, by the definition (A.4) of $W_{T}$, one also has

$$
\begin{gathered}
W_{T}(z)=W(T z)=\exp \left((T z) a^{*}-(T z)^{-} a\right) A .14 \\
(a) b
\end{gathered}
$$

then for each $z \in \mathbf{C}$

$$
(T) z
$$

with

$$
\bar{c}=12([a+d]+i[c-b]) ;-s=12([a-d]+i[c+b])
$$


defin

Remark: Notice that any $c, s$ given by (A.17) satisfy

$$
|c|^{2}-|s|^{2}=\operatorname{det}(a) b
$$

Conversely, given $c, s \in \mathbf{C}$ such that $|c|^{2}-|s|^{2}=1$, we can define $a, b, c, d$ by (A.17) and the resulting matrix is in $\mathrm{SL}(2 ; \mathbf{R})$.

Proof: Denote $\mathbf{C}^{2}(\mathbf{R})$ the real vector space

$$
\mathbf{C}^{2}(\mathbf{R})=\{\lambda(z)
$$

and $V_{0}: \mathbf{R}^{2} \rightarrow \mathbf{C}^{2}(\mathbf{R})$ the isomorphism of real vector spaces characterized by

$$
V_{0}(1)
$$

Then, if $V: \mathbf{C} \rightarrow \mathbf{R}^{2}$ is the isomorphism of Proposition A.1 and $z=x+i y$, one has

$$
V_{0} V T z=V_{0}(a) b
$$

Expressing $x, y$ in terms of $z, \bar{z}$, one finds (A.16), (A.17).

Putting together Proposition (A.2) and the identity (A.14), we obtain

$$
W_{T}(z)=\exp \left\{\left(a^{*},-a\right)(\bar{c})-s\right.
$$

Comparing (A.19) with (A.13) we finally find

$$
a_{T}=a c+a^{*} s
$$

or equivalently

$$
(a)_{T}
$$

But from (A.5),(A.7) and (A.11), it follows that the operator $U_{T}$ is characterized by the property:

$$
U_{T} a_{T} U_{T}^{*}=a
$$

or, in view of (A.20), by

$$
U_{T}^{*} a U_{T}=c a+s a^{*}
$$

Our goal is to find the operator $U_{T}$ satisfying (A.22) for given $c$ and $s$ satisfying (A.18). To this goal first notice that, in view of (A.18), there exist real numbers $r, \eta, \varphi$ such that

$$
c=\exp \{i \eta\} \cosh r=\exp \{i \eta\} c_{r} ; s=\exp \{-i \varphi\} \sinh r=\exp \{-i \varphi\} s_{r}
$$


Moreover, due to the identities

$$
\exp \left\{x a^{*} a\right\} a \exp \left\{-x a^{*} a\right\}=\exp \{-x\} a
$$

by replacing the representation $W(z)$ with the equivalent representation $W(\exp \{i \eta\} z)$, we can always suppose that $c$, in (A.22), is real, i.e., $\eta=0$ in (A.23).

Proposition A.3: Let $c, s$ be given by (A.23) with $\eta=0 ; r>0$. Then the operator $U_{T}$, characterize by $(\mathrm{A} .22)$ is given by

$$
U_{T}=\exp \left\{12\left(z a^{2}-\bar{z} a^{* 2}\right)\right\} \equiv U(z) A .25
$$

Proof: Denote $D_{z}=12\left(z a^{2}-\bar{z} a^{* 2}\right)$ and define

$$
f(t)=\exp \left\{t D_{z}\right\} a \exp \left\{-t D_{z}\right\}
$$

Then, due to the easily verified commutation relations:

$$
\left[D_{z}, a\right]=\bar{z} a^{*} ;\left[D_{z}, a^{*}\right]=z a
$$

one deduces the equation

$$
d d t(f)(t)
$$

with initial condition

whence

$$
(f)(t)
$$

For $t=1$, using (A.23) and the assumption $\eta=0$, one finds

$$
\exp \left\{D_{z}\right\} a \exp \left\{-D_{z}\right\}=c a+s a^{*}
$$

so that $U_{T}=U(z) \equiv \exp \left\{D_{z}\right\}$.

Remark: Let $z=r \exp \{i \varphi\}$ and denote $V_{t}$ the 1-parameter unitary group $V_{t}=$ $\exp \left\{i t a^{*} a\right\}$. Then one easily checks, using (A.24), that

$$
\exp \left\{D_{z}\right\}=V_{-\varphi 2} \exp \left\{D_{r}\right\} V_{\varphi 2}
$$

So we can reduce ourselves to study the operator $D_{z}$ in the case of real $z$. In several applications it is useful to know the matrix elements of the operator $\exp \left\{D_{r}\right\}=U(r)$ with respect to the coherent states in the $W$-representation. 
Proposition A.4: In the notation (A.12),(A.23),(A.25) one has

$$
<\alpha, U(r) \beta>=<0, U(r) \beta>\exp \left\{\bar{\alpha} \beta c_{r}-\bar{\alpha}^{2} s_{r} 2 c_{r}\right\} A .28
$$

Proof: Denote $f(\bar{\alpha})=<\alpha, U(r) \beta>$. Then

$$
d d \bar{\alpha} f(\bar{\alpha})=\beta c_{r}\left(1-\bar{\alpha} c_{r} \beta\right) f(\bar{\alpha}) ; f(0)=<0, U(r) \beta>
$$

Solving this equation, we find (A.27). Now put $f(r)=<0, U(r) \beta>$. Then $f(0)=1$ and

$$
d d r f(r)=12\left(\beta^{2} c_{r}^{2}-s_{r} c_{r}\right) f(r)
$$

The solution of this equation is

$$
f(r)=\exp \int_{0}^{r} 12\left(\beta^{2} c_{\tau}^{2}-s_{\tau} c_{\tau}\right) d \tau
$$

Keeping into account (A.23), the integral in (A.30) is easily evaluated and leads (A.29).

\section{References}

1. Accardi L.; Quantum Kalman filtering. Contribution to the memorial volume for the 60-th birthday of R.E.Kalman.

2. Accardi L.; Noncommutative Markov Chains with preassigned evolution: an application to the quantum theory measurement, Advanced in Mathematics, 29, (1978), 226-243.

3. Accardi L.; An outline of Quantum Probability (1990), unpublished manuscript.

4. Accardi L., Watson G.S.; Quantum random walks and coherent quantum chains. Quantum Probability and Applications IV, Springer LNM 1396, (1987), 73-88.

5. Alicki R., Frigerio A.; Quantum Poisson noise and linear quantum Boltzmann equation. preprint, March 1989.

6. Bach A.; The Simplex Structure of the Classical States of the Quantum Harmonic Oscillator. Communication Mathematical Physics, 107, (1986), 553-560.

7. Barchielli A.; Stochastic Processes and Continual Measurements in Quantum Mechanics. Preprint.

8. Bratteli O. and Robinson D.V.; Operator algebras and quantum statistical mechanics I and II, Springer Verlag, Berlin, 1981.

9. Cecchini C.; Stochastic Couplings for von Neumann Algebras. Quantum Probability and Applications III, Springer LNM 1303, (1988), 1-5.

10. Cecchini C., Petz D.; Classes of conditional expectations over von Neumann algebras. Journal of Functional Analysis 91, 1990.

11. Cecchini C., Kümmerer B.; Stochastic Transitions on Preduals of von Neumann Algebras. Quantum Probability and Applications V, Springer LNM 1442, (1990), 126-130.

12. Cecchini C., Petz D.; State Extention and a Radon-Nikodym Theorem for Conditional Expectations on von Neumann Algebras. Pacific Journal of Mathematics 138, (1989), 9-24.

13. Chen $\mathrm{Mu}-\mathrm{Fa}$, Selected topics in probability theory. Preprint Volterra N.94, 1992 
14. Fannes, M., B.Nachtergaele, R.F.Werner : Construction and study of exact ground states for a class of quantum antiferromagnets. Preprint (1989).

15. Haagerup U., A new proof of the equivalence of injectivity and hyperfiniteness for factors on a separable Hilbert space. J. Funct. Anal. 62, (1985), 160-201.

16. Hirota O., Yamazaki K., Nakagawa M., Ohya M.; Properties of error correcting code using photon pulse, Transaction of IECE Japan, E69, (1986), 917-919.

17. Hollenhorst J.N.; Quantum limits on resonant-mass gravitational-radiation detectors, Physical Review, D19, (1979), 1669-1679.

18. Kraus K.; States, effects and operations, Springer LNP N.190 (1983).

19. McEliece R.J.; Practical code for photon communication, IEEE Transaction of Information Theory, IT-27, (1981), 393-398.

20. Ohya M.; Quantum Ergodic Channels in Operator Algebras. Journal of Mathematical Analysis and Applications 84, (1981), 318-327.

21. Ohya M.; On Compound State and Mutual Information in Quantum Information Theory. IEEE Transactions of Information Theory IT-29, (1983), 770-774.

22. Ohya M.; Note on Quantum Probability. Letter al Nuovo Cimento 38, (1983), 402-404.

23. Ohya M.; State Change and Entropies in Quantum Dynamical Systems. Springer LNM 1136, (1985), 397-408 (ed) Accardi L., von Waldenfels W.

24. Ohya M.; Some Aspects of Quantum Information Theory and Their Applications to Irreversible Processes, Reports on Mathematical Physics, 27, (1989), 19-47.

25. Petz D.; Characterization of Sufficient Observation Channels. Preprint.

26. Petz D.; Sufficient Subalgebras and the Relative Entropy of States of a von Neumann Algebra. Communication Mathematical Physics, 105, (1986) 123-131.

27. Rondoni L.; Nonlinear Boltzman Maps in Classical and Quantum Probability. Quantum Probability and Related Topics VIII, World Scientific (1993)

28. Sakai S.; $C^{*}$-algebras and $W^{*}$-algebras, Springer, (1971).

29. Stoler D.; Equivalence class of minimum uncertainty packets, Physical Review, D1, (1970), 3217-3219 and D4, (1971), 1925.

30. Yuen H.P.; Two-photon coherent states of the radiation field, Physical Review, A13, (1976), 2226-2243. 